\title{
Self-peeling of impacting droplets
}

\author{
Jolet de Ruiter ${ }^{\dagger}$, Dan Soto ${ }^{\dagger}$ and Kripa K. Varanasi ${ }^{\star}$
}

\begin{abstract}
Whether an impacting droplet $^{\mathbf{1}}$ sticks or not to a solid surface has been conventionally controlled by functionalizing the target surface ${ }^{2-8}$ or by using additives in the drop ${ }^{9,10}$. Here we report on an unexpected self-peeling phenomenon that can happen even on smooth untreated surfaces by taking advantage of the solidification of the impacting drop and the thermal properties of the substrate. We control this phenomenon by tuning the coupling of the shorttimescale fluid dynamics-leading to interfacial defects upon local freezing - and the longer-timescale thermo-mechanical stresses-leading to global deformation. We establish a regime map that predicts whether a molten metal drop impacting onto a colder substrate ${ }^{11-14}$ will bounce, stick or self-peel. In many applications, avoiding adhesion of impacting droplets around designated target surfaces can be as crucial as bonding onto them to minimize waste or cleaning. These insights have broad applicability in processes ranging from thermal spraying ${ }^{12,15}$ and additive manufacturing ${ }^{16-19}$ to extreme ultraviolet lithography ${ }^{20}$.
\end{abstract}

We release molten tin droplets of millimetric size $(R=0.95 \pm 0.03 \mathrm{~mm})$ from a nozzle, such that they hit a target surface with moderate velocity ( $v=1.9 \pm 0.1 \mathrm{~m} \mathrm{~s}^{-1}$, see Methods). Figure 1a shows a comparison between the deposition and subsequent solidification of a droplet (initial temperature $T_{\mathrm{d}, 0}=240{ }^{\circ} \mathrm{C}$ ) onto a silicon wafer (top) and a glass slide with $\sim 200$ times lower thermal conductivity (bottom). While the solidified droplet (or splat) sticks to the glass surface, it unexpectedly detaches from the silicon surface: we call this phenomenon 'self-peeling' (see Fig. 1a(top) at 3 and $100 \mathrm{~ms}$, the increasing air gap between droplet and surface being a manifestation of this self-peeling).

Two main differences can be observed at this stage. First, interferometric profilometry (see Fig. 1b) confirms that the bottom of the droplet impacting on glass is perfectly flat, while it has a nearly spherical bending curvature $\kappa=16 \mathrm{~m}^{-1}$ when deposited on silicon. Second, both bottom interfaces show a texture ${ }^{21}$ formed by annular regions spaced by air ridges (insets of Fig. 1b) that act as interfacial defects. Their number is much higher for silicon than for glass. The striking contrasts in bending and defect density suggest that a balance between thermally induced stress and adhesion determines whether or not a droplet can self-peel upon solidification.

To unravel the competing phenomena, we study the dynamics of the impact using high-speed photography through a transparent surface (see Supplementary Movie 2). Immediately upon contact, micrometre-sized annular air ridges are being trapped. Along the splat radius, their height increases up to a few micrometres, and their width up to tens of micrometres (as measured by profilometry). We also extract the distance $d$ between ridges and the contact line velocity $v_{\mathrm{CL}}$ from Supplementary Movie 2, and find that the characteristic timescale $\tau=d / v_{\mathrm{CL}}$ to form these ridges increases radially. Figure $2 \mathrm{a}$ shows that the typical timescale for ridge formation, $10 \mu \mathrm{s}$, is much faster than the typical time for the droplet to completely crash $^{22}, 2 R / v \sim 1 \mathrm{~ms}$. These observations suggest that the number of ridges is set by a local competition between heat extraction-leading to solidification-and fluid motion-opposing it through local shear, mixing, and convection (see first stage of sketch in Fig. 2c). We propose that at short timescales $(<1 \mathrm{~ms}$, top row sketch of Fig. 2c), while the contact line of molten tin spreads outwards, a thin liquid layer in the immediate vicinity of the surface cools down until it forms a solid crust. At that moment, the contact line pins, while the liquid above keeps spreading on a thin air film squeezed underneath. Upon renewed touchdown of the liquid, a small air ridge remains trapped, forming the abovementioned defect structure. Another example of the fluid-thermal coupling is the existence of a texture-free central patch (see Figs $1 \mathrm{~b}$ and 2a and Supplementary Fig. 3) due to a vertical flow of hot liquid (acting as a heat supply) and a contact line with diverging ${ }^{23}$ velocity that prevents air ridge formation at early time.

Once the droplet has completely spread out, $\sim 1 \mathrm{~ms}$-forming this thin crust with air ridges that act as defects-it makes a flat pancake that solidifies (middle row of sketch in Fig. 2c) within longer timescales ( $\sim 10 \mathrm{~ms}$ in the example shown in Fig. $2 \mathrm{~b}$ ). Bending of the droplet can be tracked in real time (Supplementary Movie 2 and Fig. 2b) by measuring the spatio-temporally varying air gap $\delta$ between droplet and surface using reflection interference microscopy (RIM, see Methods). It is observed that the droplet already starts peeling-off at the time of solidification (middle row of sketch in Fig. 2c) and that its curvature is created during the homogenization of the vertical thermal gradient (up to a few $\sim 100 \mathrm{~ms}$, see bottom row of sketch in Fig. $2 \mathrm{c}$ ). If the bending stress $\sigma_{\kappa}$ overcomes adhesion stress $\sigma_{\mathrm{A}}$, the splat self-peels. Thus, microtexture formation and thermally induced bending stress are strongly separated in time, yet their combined effect determines the self-peeling behaviour of the splat. Finally, the drop cools down and thermalizes with the surroundings without noticeable change in curvature.

To predict the delamination or sticking outcome of an impacting molten droplet for a given set of parameters we assume that the droplet and surface are two semi-infinite bodies that are brought into contact ${ }^{12,24}$. In this model (see details in Supplementary Information), droplet interfacial temperature $T_{\mathrm{d} \text {,int }}$ arises as the natural parameter, is time-invariant and given by:

$$
T_{\mathrm{d}, \text { int }}=T_{\mathrm{s}, 0}+\left(T_{\mathrm{d}, 0}-T_{\mathrm{s}, 0}\right) \frac{1}{1+e_{\mathrm{s}} / e_{\mathrm{d}}} \quad \text { with } e_{i}=\left(k \rho c_{\mathrm{p}}\right)_{i}^{1 / 2}
$$

Here, $T_{\mathrm{d}, 0}$ and $T_{\mathrm{s}, 0}$ are initial droplet and substrate temperature respectively, the other relevant droplet and substrate properties being conductivity $k$, density $\rho$, and heat capacity $c_{\mathrm{p}}$. It follows from this parameterization, as shown in black in Fig. 3a, that effusivity $e_{i}$ ( $i=\mathrm{s}$ for substrate, $i=\mathrm{d}$ for droplet) is the relevant 
a

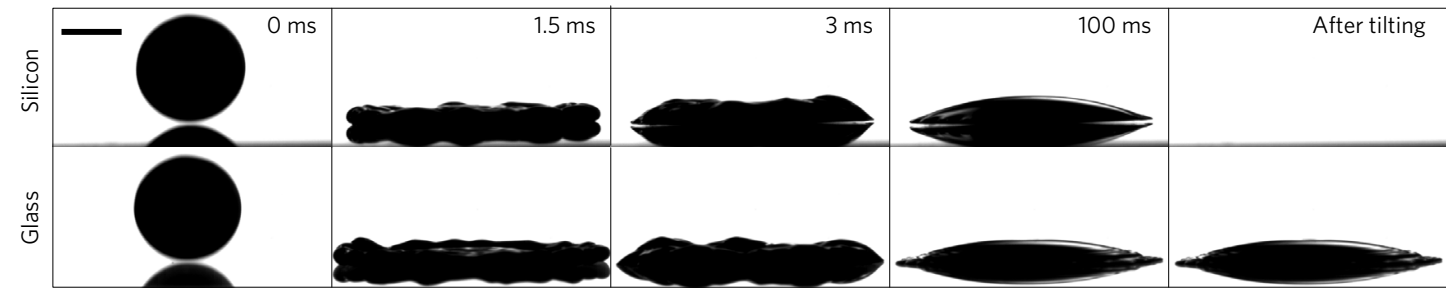

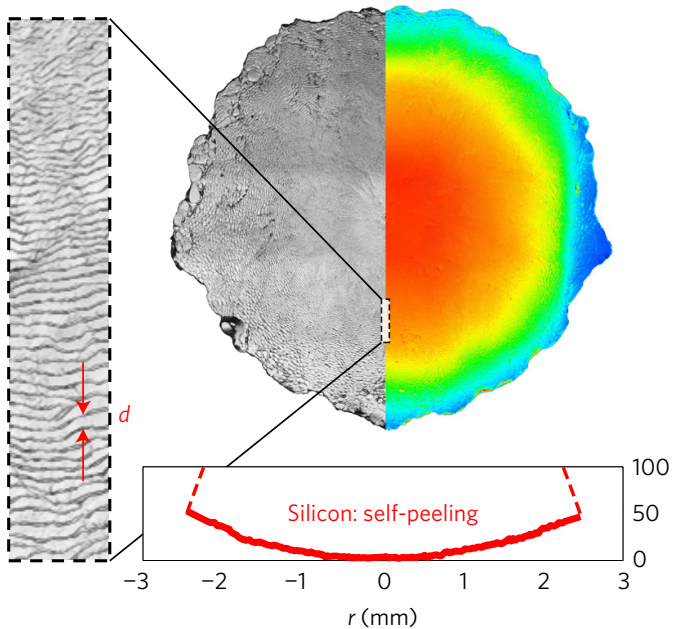
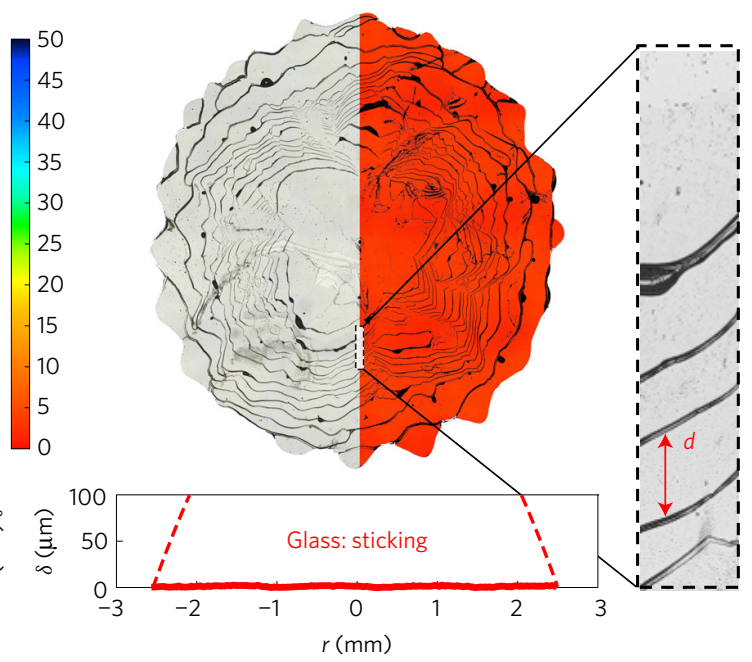

Figure 1 | Self-peeling versus sticking splats. a, Impact and solidification of a tin droplet onto a silicon wafer (top) and glass slide (bottom). The initial droplet temperature is $T_{\mathrm{d}, 0}=240^{\circ} \mathrm{C}$, and the initial surface temperature $T_{\mathrm{s}, 0}=25^{\circ} \mathrm{C}$. On silicon, the splat peels off, and slides off the surface when tilted (see Supplementary Movie 1). The onset of bending happens as early as $3 \mathrm{~ms}$, as shown by the presence of an air gap between the splat and its substrate. On glass, the splat remains attached. Scale bar: $1 \mathrm{~mm}$. Typical splat thickness (radial average) is 200 to $300 \mu \mathrm{m}$. $\mathbf{b}$, Textured bottom interface of the

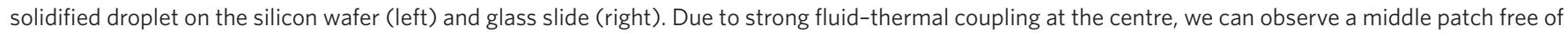
texture. Insets show a close-up view of the texture, where $d$ denotes the distance between two ridges. Silicon shows a higher ridge density compared to glass. The farther away from the centre, the bigger the distance $d$. Right half of droplet interface (in colour) shows the deflection of the interface. Below each splat, a diametral scan of the shape shows interface height $\delta$ as a function of radial position $r$.

thermal property governing our system: for large effusivity ratio $e_{\mathrm{s}} / e_{\mathrm{d}} \gg 1$, heat from the droplet can be easily transported away from the interface $\left(T_{\mathrm{d} \text {,int }} \approx T_{\mathrm{s}, 0}\right)$, while for small effusivity ratio $e_{\mathrm{s}} / e_{\mathrm{d}} \ll 1$, heat is trapped at the interface $\left(T_{\mathrm{d}, \text { int }} \approx T_{\mathrm{d}, 0}\right)$. The phase change can be taken into account using the more refined Schwarz model (in red, see Supplementary Information for details). As a consequence of the release of latent heat, this model yields a slightly higher interfacial temperature, in very good agreement with infrared thermography measurements (see Supplementary Information including Supplementary Fig. 2). The Schwarz model still predicts time-invariant $T_{\mathrm{d} \text {,int }}$ and similar dependency towards the key parameters. Since it has no analytical solution, the simpler conduction model remains extremely valuable to predict parameters' dependency. We deliberately ignore more complex dynamics due to coupling of fluid dynamics, solid mechanics, and heat transfer-for example, liquid convection inside the splat, overall splat cooling by convection, and delamination that changes the thermal boundary condition-that can be addressed only by using a full numerical simulation.

Predicting interfacial temperature is key since it is expected to play a major role both on defect density (related to adhesion ${ }^{25}$, see Fig. 4c) and thermal stresses (related to bending ${ }^{26,27}$ ). As droplet interfacial temperature $T_{\mathrm{d} \text {,int }}$ decreases, it is easier to solidify the spreading liquid, so we expect defect distance $d$ to decrease as well. Indeed, since Fig. 3a predicts a lower interfacial temperature for silicon wafer compared to glass, we should observe an increased defect density for the silicon substrate, as confirmed in Fig. 1b. It can be deduced from this enhanced defect density on silicon that its adhesion to the splat should be in turn lower (compared to glass). Regarding bending, if we approximate the splat by a free thin metal disk with a linear through-the-thickness temperature gradient $\Delta T$, thin plate theory ${ }^{26}$ predicts a spherical curvature $\kappa \sim \alpha(\Delta T / h)$ due to thermal expansion of the tin upon cooling ( $\alpha$, linear expansion coefficient, $h$, thickness). Maximum thermal gradient across the solid splat-setting the maximum bending that we can observe eventually-occurs at the time of full solidification (upon which the model is still valid, see Methods). If we assume $\Delta T \sim T_{\text {melt }}-T_{\text {d,int }}$ and use $\kappa$ as an indirect measurement of the thermal stresses, we expect the stress to increase with decreasing interfacial temperature. This is confirmed in Fig. $3 \mathrm{~b}$ when varying either $T_{\mathrm{s}, 0}$ (red markers) or $T_{\mathrm{d}, 0}$ (blue markers) and calculating the corresponding $T_{\mathrm{d}, \text { int }}$ using the Schwarz model. Symmetrically, for sufficiently high $T_{\mathrm{d} \text {,int }}$, the curvature drops to zero as adhesion stress $\sigma_{\mathrm{A}}$ overcomes thermally induced stress $\sigma_{\kappa}$ and the splat sticks to the surface (squares). For even higher interfacial temperature the droplet remains liquid and bounces off (star). Based on these observations, we define a critical initial surface temperature $T_{\mathrm{s}, 0}^{*}$, or critical initial droplet temperature $T_{\mathrm{d}, 0}^{*}$, for which the droplet interface remains above the solidification temperature and the whole droplet remains liquid. In this case the Schwarz model converges to the conduction model-with analytical solution in equation (1) - and introducing $T_{\mathrm{d} \text {,int }}=T_{\text {melt }}$ leads to:

$$
T_{\mathrm{d}, 0}^{*}=T_{\text {melt }}+\frac{e_{\mathrm{s}}}{e_{\mathrm{d}}}\left(T_{\text {melt }}-T_{\mathrm{s}, 0}\right)
$$

or

$$
T_{\mathrm{s}, 0}^{*}=T_{\text {melt }}-\frac{e_{\mathrm{d}}}{e_{\mathrm{s}}}\left(T_{\mathrm{d}, 0}-T_{\text {melt }}\right)
$$



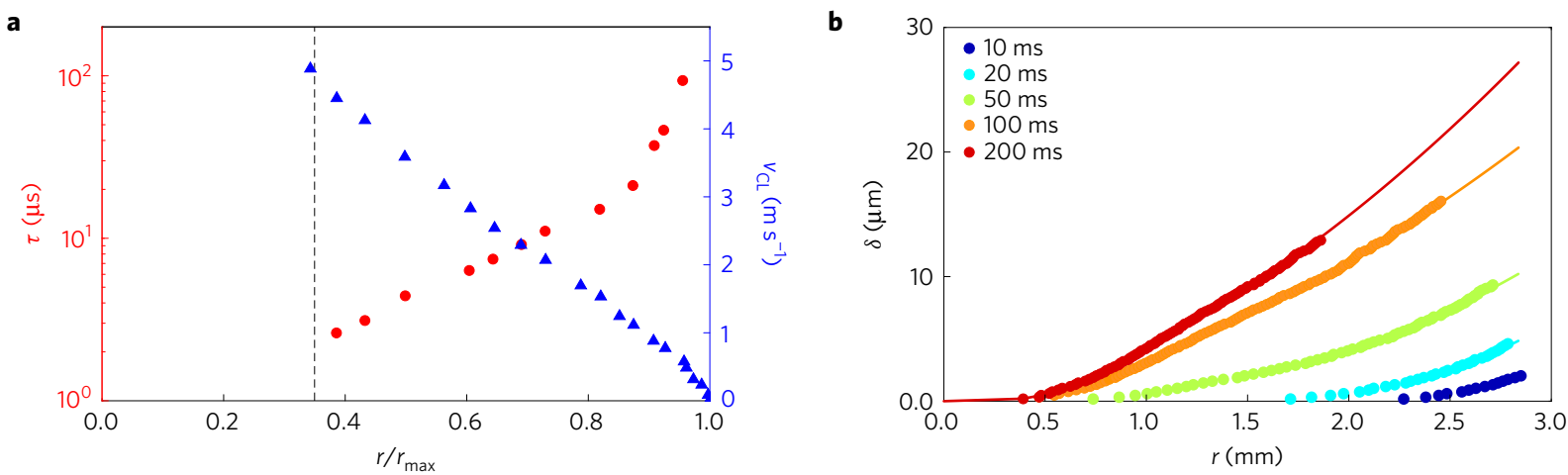

c
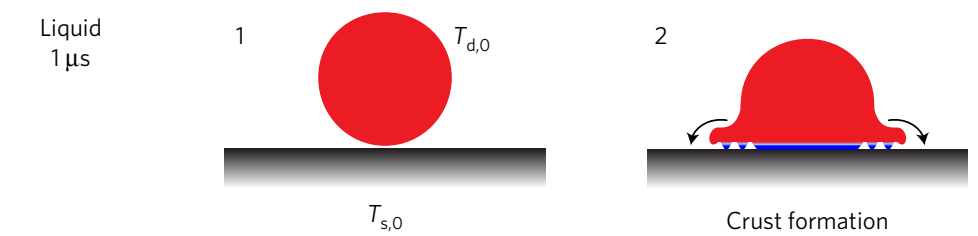

3

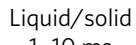

4 1-10 ms

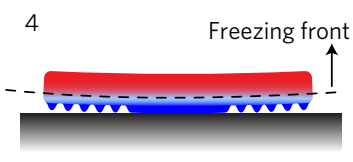

5

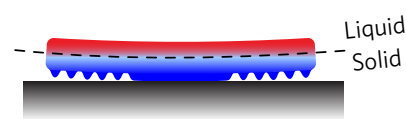
Solid
-1,000 ms

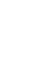

6
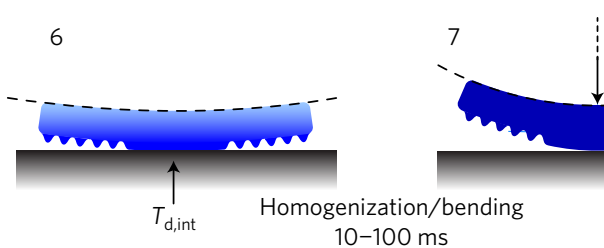

$10-100 \mathrm{~ms}$

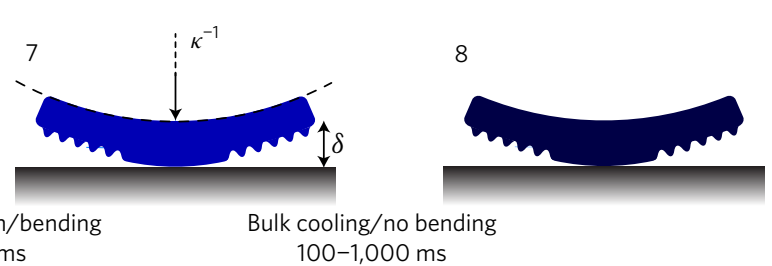

Figure 2 | Dynamics. a, Characteristic ridge formation timescale $\tau=d / v_{\mathrm{CL}}$ (circles) and contact line velocity $v_{\mathrm{CL}}$ (triangles), as a function of normalized radial position, $r / r_{\max }$. For $r / r_{\max }<0.35$, the interface is free of textures due to the combination of both diverging contact line speed and vertical flow of hot liquid happening at the axis of symmetry. b. Splat bending dynamics showing deflection $\delta$ as function of radial position $r$ at different times. Most bending happens when the solidified droplet homogenizes in temperature (between 10 and $100 \mathrm{~ms}$ ). Circles: data obtained through the RIM imaging technique (resolution limited to $\sim 1^{\circ}$ slope). Solid line: data extrapolation to whole depth (see Methods). a,b correspond to impact onto a zinc selenide surface with $T_{\mathrm{d}, 0}=240^{\circ} \mathrm{C}$ and $T_{\mathrm{s}, 0}=25^{\circ} \mathrm{C}$. c. Sketch based on the droplet phase. During the liquid phase (up to $1 \mathrm{~ms}$, steps 1 to 3 ) the interface texture is formed as a result of the local coupling between fluid dynamics and heat transfer. During the solid-liquid coexistence ( 1 to $10 \mathrm{~ms}$, steps 4 to 5 ) the onset of bending occurs. The homogenization of the vertical thermal gradient (up to hundreds of milliseconds, steps 6 to 7 ) leads to bending curvature. The splat finally cools down as a bulk (up to timescales of seconds, steps 7 to 8) without further bending happening. Red denotes liquid tin and blue denotes solid tin (darker blue indicates lower temperature).

With these few ingredients, we can now summarize in Fig. 4a,b the overall impact outcome in a three-region phase diagram: a regime of interfacial freezing (or interfacial melting, not treated experimentally) where the drop undergoes phase change (blue areas) and two more familiar regimes consisting of a liquid droplet never freezing (red area: $T_{\mathrm{d}, 0}>T_{\mathrm{d}, 0}^{*}$ ) and a solid droplet never melting (pale blue area, see Supplementary Information). Within the interfacial freezing regime, as we approach $T_{\mathrm{d}, 0}^{*}$, adhesion stresses increase-due to lower defect density-at the same time that thermal stresses decrease-due to smaller temperature gradients. As a consequence, there will be a crossover point below which the splat self-peels $\left(\sigma_{\kappa}>\sigma_{\mathrm{A}}\right)$ and above which it sticks $\left(\sigma_{\mathrm{A}}>\sigma_{\kappa}\right)$. To validate our model, we performed experiments with varying surface temperature (Fig. 4a) and surface effusivity (Fig. 4b), and recorded the possible deposition behaviour: liquid recoil (stars), sticking (squares) and self-peeling (circles). The corresponding results highlight the ubiquitous presence of self-peeling and liquid recoil with a sticking region in between, and validate the choice of interfacial temperature as a parameter allowing a simple parameterization of our system.
Although the broadening of the sticking regime above $T_{\mathrm{d}, 0}^{*}$ surpasses the parameterization, we experimentally characterize this phenomenon by studying the existence of a central re-molten patch (see glass, fused silica and central region of quartz and zinc selenide in Fig. 4c). Its texture is made of bubbles resulting from the contraction of the air ridges trapped in the interfacial crust. Its presence is the signature of the transient formation of an initial crust (see Supplementary Movie 3) that is subsequently re-melted, typically hundreds of microseconds later. This phenomenon is restricted to a region close to $T_{\mathrm{d}, 0}^{*}$ (see Fig. $4 \mathrm{c}$ and Supplementary Figs 4 and 5). Indeed, if the initial droplet temperature is too high, $T_{\mathrm{d}, 0} \gg T_{\mathrm{d}, 0}^{*}$, the crust never forms (recoil regime) and if it is too low, $T_{\text {melt }}<T_{\mathrm{d}, 0} \ll T_{\mathrm{d}, 0}^{*}$, the liquid flowing on top of the crust does not have enough energy to re-melt it (interfacial freezing regime). In between, extra sticking behaviour exists (extending the initial prediction above $T_{\mathrm{d}, 0}^{*}$ ) due to the transient formation of a crust not able to completely re-melt, particularly at the outermost radial portion of the splat where there is an absence of hot liquid above it. As a consequence, just before full recoil can happen, we systematically observe de-wetting starting from the centre 

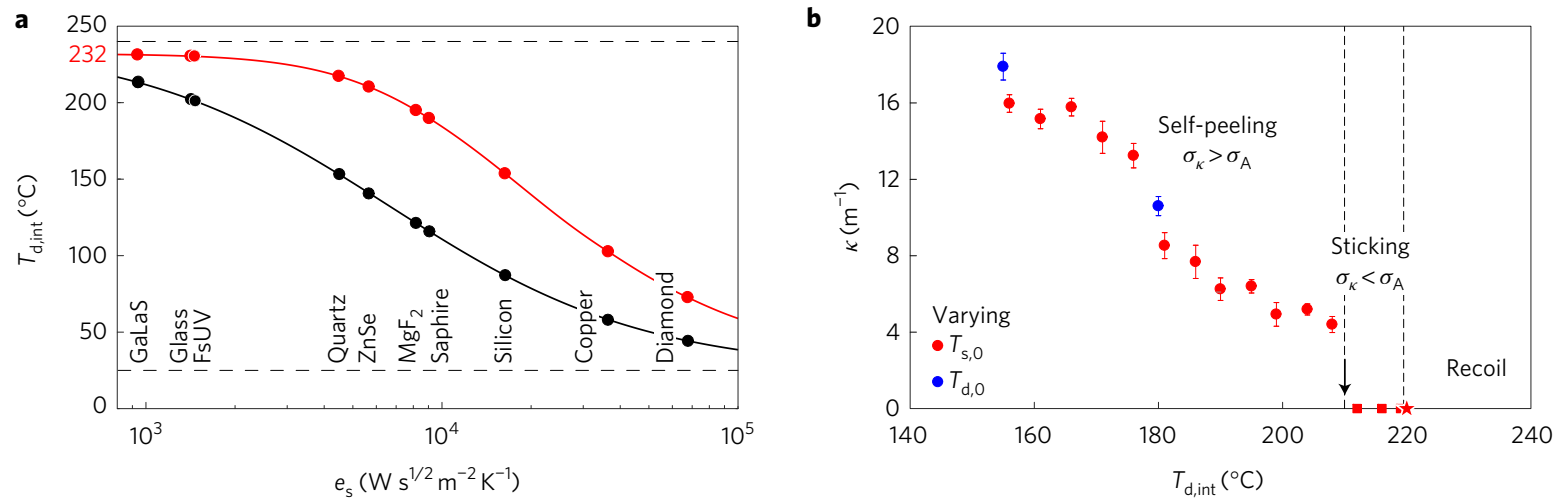

Figure $\mathbf{3}$ | Splat interfacial temperature and bending. a, Droplet interfacial temperature $T_{\text {d,int }}$ as a function of surface effusivity $e_{s}$. Circular markers represent the experimentally tested surfaces, with $T_{\text {d,int }}$ calculated using the conduction model (black) or the Schwarz model (red). Surface temperature $T_{\mathrm{s}, 0}=25^{\circ} \mathrm{C}$ and droplet temperature $T_{\mathrm{d}, 0}=240^{\circ} \mathrm{C}$ are indicated by black dashed lines. Effusivity ratios $e_{\mathrm{s}} / e_{\mathrm{d}}$ vary over two orders of magnitude: $0.1<e_{s} / e_{d}<10 . \mathbf{b}$, Splat bending curvature $\kappa$ as a function of interfacial temperature $T_{\text {d,int }}$ (using Schwarz model) on a silicon wafer. We experimentally vary the initial surface temperature $T_{\mathrm{s}, 0}$ (red circles; constant $T_{\mathrm{d}, 0}=240^{\circ} \mathrm{C}$ ) or the initial droplet temperature $T_{\mathrm{d}, 0}$ (blue circles; constant $T_{\mathrm{s}, 0}=25^{\circ} \mathrm{C}$ ). Experimental data points have a standard deviation based on four to six repeated experiments. For low interfacial temperature, bending stresses overcome adhesion stresses, $\sigma_{\kappa}>\sigma_{\mathrm{A}}$ and the splat bends (circles). For increasing interfacial temperature, $\sigma_{\kappa}<\sigma_{\mathrm{A}}$ and the splat sticks to the surface (squares). For even higher interfacial temperature reaching $T_{\text {melt }}$, the droplet remains liquid and recoils (star).

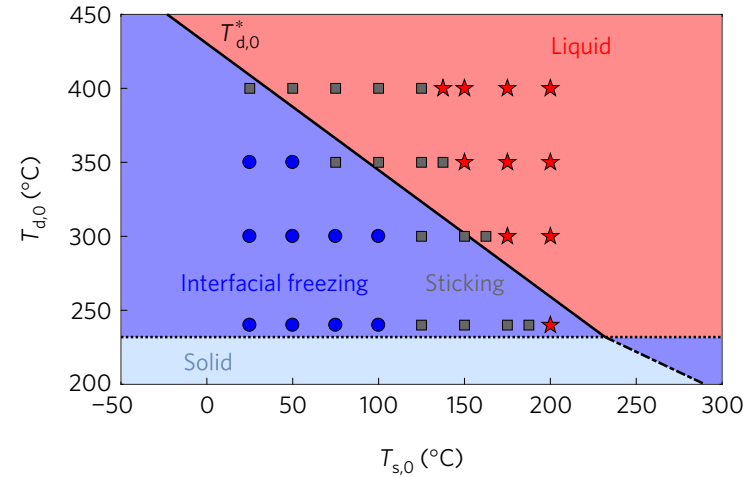

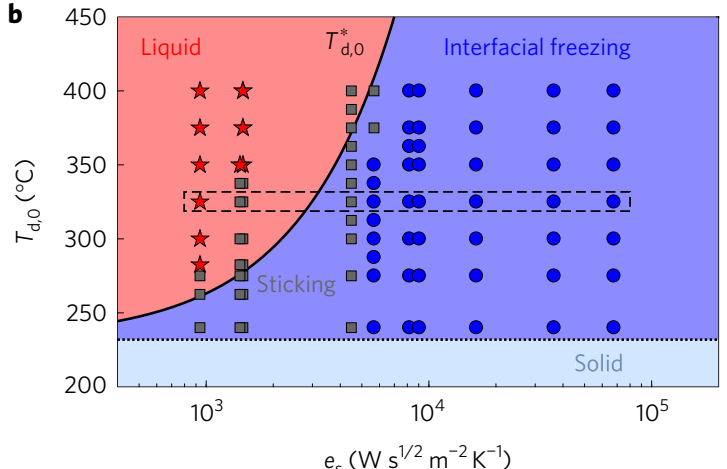

c

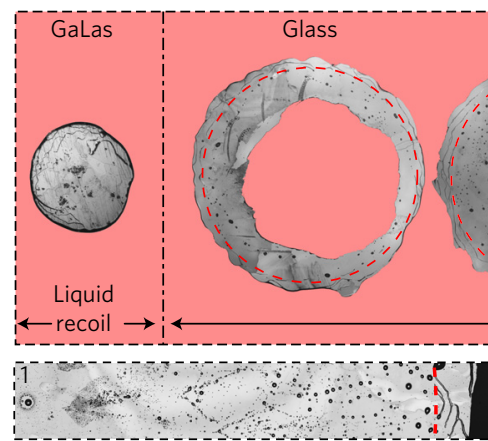

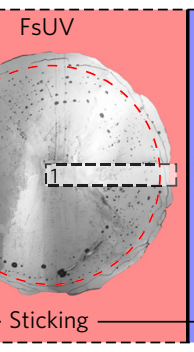
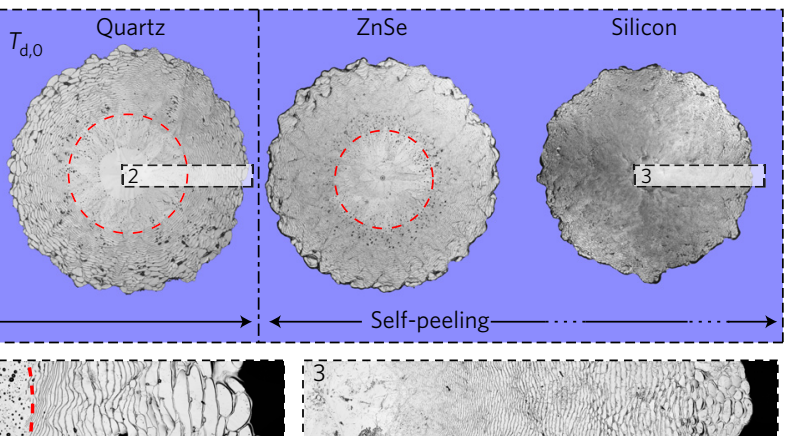

10

Figure $\mathbf{4}$ | Phase diagram of splat outcomes. Phase diagrams (a,b) and bottom interface view showing interfacial textures (c). a,b, Markers show experimental outcome after impact (red stars: liquid recoil, grey squares: sticking, blue circles: self-peeling) as a function of both initial droplet temperature $T_{\mathrm{d}, 0}$ and either initial zinc selenide surface temperature $T_{\mathrm{s}, 0}$ (with $e_{\mathrm{s}}=5.7 \times 10^{3} \mathrm{~W} \mathrm{~s}^{1 / 2} \mathrm{~m}^{-2} \mathrm{~K}^{-1}$ ) (a) or surface effusivity $e_{\mathrm{s}}$ at constant surface temperature $T_{\mathrm{s}, 0}=25^{\circ} \mathrm{C}(\mathbf{b})$. Solid black line satisfies equation (2) (valid for both conduction and Schwartz model) and defines the transition temperature $T_{\mathrm{d}, 0}^{*}$ between a fully liquid droplet (red area) and an interfacial freezing (either self-peeling or sticking) regime (blue area). Dotted black line sets the limit towards the trivial fully solid impact regime (pale blue area). c, Selection of snapshots (top row, corresponding to selection box in $\mathbf{b}$ ) and microtextures (bottom row, corresponding to numbered selection boxes) for the various surfaces with constant $T_{\mathrm{d}, 0}=325^{\circ} \mathrm{C}$ and $T_{\mathrm{s}, 0}=25^{\circ} \mathrm{C}$. Microtextures show (right to left) the decrease of defect density and the increase of re-melted centre area (red dashed boundary). Transition from sticking to bouncing happens after a stage of central de-wetting into the outer ring above $T_{\mathrm{d}, 0}^{*}$.

leading to liquid accumulation on the pinned ring as seen for glass in Fig. 4c.

We introduce a new approach to control droplet adhesion to the substrate based on thermal properties of the solid, rather than surface functionalization. Even if a droplet of molten liquid impacting on a surface can initially stick, self-peeling can occur upon solidification and result in detachment. We identify that the coupling of early-time defect formation to late-time solid mechanics governs this phenomenon. By using a simple model based on the interfacial temperature, and balancing adhesion with bending 
stresses, we are able to define and capture the possible outcome regimes: bouncing (liquid or solid), sticking and self-peeling. We expect that this approach can be extended to other liquids ${ }^{28-30}$ (water, wax, thermoplastics) and other freezing applications where adhesion must be controlled, such as powder metallurgy, inkjet printing and de-icing.

\section{Methods}

Methods, including statements of data availability and any associated accession codes and references, are available in the online version of this paper.

Received 24 February 2017; accepted 9 August 2017; published online 11 September 2017

\section{References}

1. Josserand, C. \& Thoroddsen, S. T. Drop impact on a solid surface. Annu. Rev. Fluid Mech. 48, 365-391 (2016).

2. Quéré, D. Non-sticking drops. Rep. Prog. Phys. 68, 2495-2532 (2005).

3. Gauthier, A., Symon, S., Clanet, C. \& Quéré, D. Water impacting on superhydrophobic macrotextures. Nat. Commun. 6, 8001 (2015).

4. Liu, Y. et al. Pancake bouncing on superhydrophobic surfaces. Nat. Phys. 10, 515-519 (2014).

5. Lu, Y. et al. Robust self-cleaning surfaces that function when exposed to either air or oil. Science 347, 1132-1135 (2015).

6. Schutzius, T. M. et al. Spontaneous droplet trampolining on rigid superhydrophobic surfaces. Nature 527, 82-85 (2015).

7. Deng, X., Mammen, L., Butt, H.-J. \& Vollmer, D. Candle soot as a template for a transparent robust superamphiphobic coating. Science 335, 67-70 (2012).

8. Bird, J. C., Dhiman, R., Kwon, H.-M. \& Varanasi, K. K. Reducing the contact time of a bouncing drop. Nature 503, 385-388 (2013).

9. Bergeron, V., Bonn, D., Martin, J. Y. \& Vovelle, L. Controlling droplet deposition with polymer additives. Nature 405, 772-775 (2000).

10. Damak, M., Mahmoudi, S. R., Hyder, M. N. \& Varanasi, K. K. Enhancing droplet deposition through in-situ precipitation. Nat. Commun. 7, 12560 (2016)

11. Schiaffino, S. \& Sonin, A. A. Molten droplet deposition and solidification at low Weber numbers. Phys. Fluids 9, 3172-3187 (1997).

12. Aziz, S. D. \& Chandra, S. Impact, recoil and splashing of molten metal droplets. Int. J. Heat Mass Transfer 43, 2841-2857 (2000).

13. Attinger, D., Zhao, Z. \& Poulikakos, D. An experimental study of molten microdroplet surface deposition and solidification: transient behavior and wetting angle dynamics. J. Heat Transfer 122, 544-556 (2000).

14. Pasandideh-Fard, M., Chandra, S. \& Mostaghimi, J. A three-dimensional model of droplet impact and solidification. Int. J. Heat Mass Transfer 45, 2229-2242 (2002).

15. Mostaghimi, J., Chandra, S., Ghafouri-Azar, R. \& Dolatabadi, A. Modeling thermal spray coating processes: a powerful tool in design and optimization. Surf. Coat. Technol. 163, 1-11 (2003).
16. Skylar-Scott, M. A., Gunasekaran, S. \& Lewis, J. A. Laser-assisted direct ink writing of planar and 3D metal architectures. Proc. Natl Acad. Sci. USA 113, 6137-6142 (2016)

17. Truby, R. L. \& Lewis, J. A. Printing soft matter in three dimensions. Nature 540, 371-378 (2016).

18. Wang, C.-H., Tsai, H.-L., Wu, Y.-C. \& Hwang, W.-S. Investigation of molten metal droplet deposition and solidification for 3D printing techniques. J. Micromech. Microeng. 26, 95012 (2016).

19. Ladd, C., So, J.-H., Muth, J. \& Dickey, M. D. 3D printing of free standing liquid metal microstructures. Adv. Mater. 25, 5081-5085 (2013).

20. Wagner, C. \& Harned, N. EUV lithography: lithography gets extreme. Nat. Photon. 4, 24-26 (2010).

21. Thoroddsen, S. T., Takehara, K. \& Etoh, T. G. Bubble entrapment through topological change. Phys. Fluids 22, 51701 (2010).

22. Lagubeau, G. et al. Spreading dynamics of drop impacts. J. Fluid Mech. 713, 50-60 (2012).

23. Eddi, A., Winkels, K. G. \& Snoeijer, J. H. Short time dynamics of viscous drop spreading. Phys. Fluids 25, 13102 (2013).

24. Carslaw, H. S. \& Jaeger, J. C. Conduction of Heat in Solids (Oxford Univ. Press, 1986).

25. Subramanyam, S. B., Rykaczewski, K. \& Varanasi, K. K. Ice adhesion on lubricant-impregnated textured surfaces. Langmuir 29, 13414-13418 (2013).

26. Bower, A. F. Applied Mechanics of Solids (CRC Press, 2009).

27. Sundaram, S., Lipkin, D. M., Johnson, C. A. \& Hutchinson, J. W. The influence of transient thermal gradients and substrate constraint on delamination of thermal barrier coatings. J. Appl. Mech. 80, 11002 (2013).

28. Ghabache, E., Josserand, C. \& Séon, T. Frozen impacted drop: from fragmentation to hierarchical crack patterns. Phys. Rev. Lett. 117, 074501 (2016).

29. Beesabathuni, S. N., Lindberg, S. E., Caggioni, M., Wesner, C. \& Shen, A. Q. Getting in shape: molten wax drop deformation and solidification at an immiscible liquid interface. J. Colloid Interface Sci. 445, 231-242 (2015).

30. Galliker, P. et al. Direct printing of nanostructures by electrostatic autofocussing of ink nanodroplets. Nat. Commun. 3, 890 (2012).

\section{Acknowledgements}

We acknowledge funding from Alstom. J.d.R. acknowledges a Rubicon fellowship from the Netherlands Organisation for Scientific Research (NWO). We thank F. Mugele from the University of Twente for using their reflection interference microscopy set-up.

\section{Author contributions}

J.d.R., D.S. and K.K.V. designed the research. J.d.R. and D.S. performed the research. J.d.R., D.S. and K.K.V. analysed the data and wrote the manuscript.

\section{Additional information}

Supplementary information is available in the online version of the paper. Reprints and permissions information is available online at www.nature.com/reprints. Publisher's note: Springer Nature remains neutral with regard to jurisdictional claims in published maps and institutional affiliations. Correspondence and requests for materials should be addressed to K.K.V.

\section{Competing financial interests}

The authors declare no competing financial interests. 


\section{Methods}

Droplet impact experiments. To create molten tin droplets, tin shot (Sigma-Aldrich) is heated to a temperature above $T_{\text {melt }}=231.9^{\circ} \mathrm{C}$ in a stainless steel cavity surrounded by a resistive heater ${ }^{31}$. The liquid tin temperature is measured near the nozzle and is varied between 240 and $400( \pm 1)^{\circ} \mathrm{C}$. Droplets are shot through a sapphire nozzle $(D=885 \mu \mathrm{m}$ ring jewel bearing from SwissJewel Company) using a pressure pulse generated by a solenoid valve (ASCO 411 Series, $15 \mathrm{VDC} 2.0 \mathrm{~W}$ ). The pulse is tuned using a pulse generator (Quantum Composers Plus 9518), varying the pressure from 65 to 75 psi and pulse width from 13 to $17 \mathrm{~ms}$, to obtain a single jetted droplet of millimetric size, $R=0.95 \pm 0.03 \mathrm{~mm}$. The temperature of the droplet at impact $T_{\mathrm{d}, 0}$ is assumed to be similar to the liquid tin temperature; cooling during flight due to flow convection is estimated to be smaller than $5^{\circ} \mathrm{C}$. The thermal properties of tin are shown in Supplementary Table 2. To prevent tin oxide skin formation, the droplets are formed within a closed chamber that is purged with nitrogen ${ }^{32}$. We verified that droplets are spherical upon impact (in contrast to oxidized droplets that show a characteristic tail). The droplets spread to a pancake-like shape since their impact Weber number We $=\rho R v^{2} / \sigma=48$ ( $\rho$ : liquid density; $\sigma$ : surface tension) is well below the splashing threshold.

We use a series of target surfaces to vary the surface effusivity $e_{\mathrm{s}}$ over two orders of magnitude, from $10^{3}$ to $10^{5} \mathrm{Ws}^{1 / 2} \mathrm{~m}^{-2} \mathrm{~K}^{-1}$. Crystalline optical materials are obtained from Crystran, copper from McMaster Carr (super conductive 101 that was polished by Princeton Scientific Corp. to an r.m.s. roughness of $2.6 \mathrm{~nm}$ ), and glass slides from Fisher Scientific. Since roughness is at maximum a few nanometres for all surfaces, we expect splat-substrate adhesion to arise from dispersive adhesion. Since the drop gradually detaches from the perimeter inwards, bending stresses originating from differential shrinking between top and bottom of the splat (as for splats with mechanical interlocking bonded central area ${ }^{33}$ ) are expected to be negligible. Since all substrates have a crystalline form (except the copper), they have a high surface energy-of the order of $1 \mathrm{~J} \mathrm{~m}^{-2}$ (versus $30 \mathrm{~mJ} \mathrm{~m}^{-2}$ for low-energy surfaces such as plastics and composites). Adhesion between splat and substrate is set by surface energy and the area of contact. Since the latter varies strongly between surfaces, due to strong variations of defect density at the splat-substrate interface, we expect it to be the dominant parameter, while relatively small variations in surface energy can be neglected.

Thermal properties of all target surfaces are shown in Supplementary Table 1. In addition to surface effusivity, the surface temperature $T_{\mathrm{s}, 0}$ is varied between 25 and $200( \pm 1){ }^{\circ} \mathrm{C}$. Changing surface properties $e_{\mathrm{s}}$ and $T_{\mathrm{s}, 0}$ gives a more controlled mechanism to change the interfacial temperature (equation (1)) than changing the temperature $T_{\mathrm{d}, 0}$ of the droplet. (Indeed, the droplet being liquid, its thermal properties are strongly coupled with fluid dynamic properties. A variation of the liquid temperature is expected to directly impact interfacial temperature, but may indirectly affect it as well through the modification of the flow dynamics). Surface thickness $L$ is chosen such that the characteristic timescale for diffusion $\tau_{\mathrm{d}}=L^{2} / \alpha_{\text {diff }}>100 \mathrm{~ms}$ is larger than the timescale for droplet solidification $\left(\alpha_{\text {diff }}\right.$ : thermal diffusivity of the material). This implies that all target surfaces except diamond can be considered as semi-infinite bodies for the purpose of interfacial temperature calculations. It should be noted that our tin splats have a $\tau_{\mathrm{d}} \approx 10 \mathrm{~ms}$, after which finite effects start to play a role. Most importantly, we can consider our semi-infinite body model as a good candidate up to the freezing time (order $10 \mathrm{~ms}$ ) when the maximum cross-thickness temperature gradient-that determines the final splat bending curvature-is set.

Dynamic analysis. The dynamics of droplet impingement and solidification are recorded using high-speed photography (Photron Fastcam SA1.1) at up to 20,000 frames per second. Recording from the side yields the evolution of the overall drop shape and an estimate of the bulk solidification time. Recording from the bottom yields the dynamics of defect formation and lift-off; this requires a transparent surface (thus excluding silicon and copper). In addition, observing the dynamics of lift-off relies on interference of reflections from the target surface and the droplet that yield a typical fringe pattern (see Supplementary Movie 2) from which the air gap between droplet and surface can be deducted. We use reflection interference microscopy (RIM set-up: see ref. 34) with a wavelength $\lambda=572 \mu \mathrm{m}$, leading to a vertical spacing of approximately $\lambda / 4=143 \mu \mathrm{m}$ between subsequent fringes. The contrast of the RIM technique is a function of the reflection coefficients of the tin and the target surface; in our set of surfaces, zinc selenide gives the highest contrast, and is used for demonstration in Supplementary Movie 2 and Fig. 2. The depth of view is set by both $\lambda / 4$ and the lateral resolution of the microscope objective, which limits visibility to interface slopes of $\lesssim 1^{\circ}$ (ending of data points in Fig. 2b).

Equilibrium curvature. 'Self-peeling' splats can be gently brushed-off from the target substrate using a cotton tip, which leaves their bent shape undisturbed. Their equilibrium curvature is visualized by means of an optical profilometer using white light (Zygo) or laser (Keyence VK-x250 Laser Microscope) interferometry. The obtained shape is fitted with a spherical cap to extract the curvature $\kappa$. Sphericity is confirmed visually and by agreement (within typically $7 \%$ ) of $\kappa$ fitted for either the full splat or the centre region $(r=1.5 \mathrm{~mm})$. Deviations are mainly attributed to slight folding of splats, or slight flattening in the stickier central patch. An average curvature is determined based on four to six repeated experiments per experimental condition.

Data availability. The data that support the plots within this paper and other findings of this study are available from the corresponding author upon request.

\section{References}

31. Cheng, S. X., Li, T. \& Chandra, S. Producing molten metal droplets with a pneumatic droplet-on-demand generator. J. Mater. Process. Technol. 159, 295-302 (2005).

32. Xu, Q., Brown, E. \& Jaeger, H. M. Impact dynamics of oxidized liquid metal drops. Phys. Rev. E 87, 43012 (2013).

33. Xue, M., Chandra, S. \& Mostaghimi, J. Investigation of splat curling up in thermal spray coatings. J. Therm. Spray Technol. 15, 531-536 (2006).

34. de Ruiter, J., Oh, J. M., van den Ende, D. \& Mugele, F. Dynamics of collapse of air films in drop impact. Phys. Rev. Lett. 108, 074505 (2012). 